\title{
Teaching Einsteinian gravity in Italian primary school ${ }^{\dagger}$
}

\author{
Sara Mattiello *, Matteo Luca Ruggiero *, Matteo Leone
}

Publisher's Note: MDPI stays neutral with regard to jurisdictional claims in published maps and institutional affiliations.

\section{(c) (i)}

Copyright: (c) 2021 by the authors. Submitted for possible open access publication under the terms and conditions of the Creative Commons Attribution (CC BY) license (http://creativecommons.org/licenses /by/4.0/).

\begin{abstract}
Why, modern physics is, still today, more than 100 years after its birth, privilege of an elite of scientists and unknown for the great majority of citizens? The answer is simple, since modern physics is in general not present in the standard physics curricula, except for some general outlines, in the final years of some secondary schools. But, is it possible to teach modern physics in primary school? Is it effective? And, also, is it funny for the students? We firmly believe that general relativity and quantum mechanics are among the greatest intellectual achievements of mankind and, hence, their knowledge and appreciation should not be reserved to an elite of researchers; consequently, we are convinced that, in the spirit of Einstein First project, students of all ages need to be exposed to the current paradigm of physics. Starting from these premises, in this talk we report the results of an intervention performed in the last year of Italian primary school in which we introduced some concepts of the theory of relativity, such as the role of the reference frame, the velocity addition law, the peculiar characteristics of light propagation, the role of simultaneity and, eventually, gravitational interactions according to the Einsteinian picture.
\end{abstract}

Keywords: einsteinian physics, relativity, primary school. 